\title{
DEVELOPMENT OF A WIND OBSERVATION SYSTEM USING PHOTOGRAMMETRY
}

\author{
Hideaki KAWASAKI $^{\text {a, }}$, Toshio KOIZUMI ${ }^{\text {b }}$, Kazuhiro MATSUDA ${ }^{\mathrm{c}}$ \\ ${ }^{a}$ Bureau of Port and Harbor, Tokyo Metropolitan Government, 3-9-56,Kounan,Minatoku,Tokyo 108-0075,Japan \\ E-mail: Hideaki_Kawasaki@member.metro.tokyo.jp \\ ${ }^{\mathrm{b}}$ Department of Architecture and Civil Engineering, Chiba Institute of Technology, \\ 2-17-1, Tsudanuma, Narashino-shi, chiba 275-0016, Japan \\ E-mail: koizumi.toshio@it-chiba.ac.jp \\ ${ }^{\mathrm{c}}$ Kaihatsu-toranomon concultant Co.,Ltd, 3-20-6,Minamiotsuka,Toshimaku,Tokyo 170-0005,Japan
}

Commission V, WG V/1

KEY WORDS: Wind-flow, Photogrammetry, Inertial photogrammetry unit, Photo-theodolite, Digital still camera, Path of particle

\begin{abstract}
:
Quantitative understanding of wind flow near the ground surface in all three dimensions onsite is extremely important for wind research.The objective of this research is to develop a system to quantitatively measure wind flow near the ground surface in both the horizontal and vertical directions (in other words, wind flow in three dimensions) using inertial photographic surveying or phototheodolite. In this method, a balloon (a no-lift balloon) with the same relative weight as air and soap bubbles were released as tracers. Their movements were captured using stereo-photography and single photographs, and then the path of particles was analyzed in three dimensions. For this document, wind flow near tall buildings, conical vortices flow on the rooftops of buildings and wind flow crossing over an embankment were measured. The results of the testing proved that the measurement methods were effective.
\end{abstract}

\section{INTRODUCTION}

Quantitative understanding of wind flow near the ground surface in all three dimensions onsite is extremely important for wind research.The objective of this research is to develop a system to quantitatively measure wind flow near the ground surface in both the horizontal and vertical directions (in other words, wind flow in three dimensions) using photogrammetry. In this method, a balloon (a no-lift balloon) with the same relative weight as air and soap bubbles were released as tracers. Their movements were captured using stereo-photography and single photographs, and then the path of particles was analyzed in three dimensions.

In this research, two photogrammetry devices were manufactured. One device was a digital still camera with an inertial measurement unit attached. (In this paper, this device is referred to as an inertial photogrammetry unit.) This device does not require a control point because the exterior orientation parameters can be measured from the inertial measurement unit. In addition, the tracer movement can be followed and photographed during movement. Along with the single camera, this device is equipped with a stereo camera with a camera baseline length of $2 \mathrm{~m}$. The other device was a photo-theodolite equipped with a digital still camera. The device was manufactured so that it could photograph continuously. For measurement of wind flow near tall buildings, a spherical balloon with a diameter of $40 \mathrm{~cm}$ was released as a tracer. As the balloon moved, it was tracked using the single camera of the inertial photogrammetry unit. Photographs were taken at arbitrary intervals, and the path of particles was measured using inertial single photogrammetry. For measurement of wind flow crossing over an embankment, soap bubbles with diameters of between 10 and $20 \mathrm{~cm}$ were continuously released. As the bubbles moved, they were tracked using the stereo camera of the inertial photogrammetry unit. Images were taken at a rate of 6.5 frames per second to measure the path of particles. Conical vortices form on the rooftops of buildings and apply negative pressure to the roofs, which causes roofing material to peel. In this paper, soap bubbles were continuously released on the rooftop of buildings, photos were taken using the phototheodolite, and the occurrences of conical vortices and their movements could be acquired in detail.

\section{PHOTOGRAMMETRY INSTRUMENTS USED IN THIS STUDY}

\subsection{Inertial Single Photogrammetry Instrument}

The instrument is shown in Figure.1. The instrument is set in a back pack type carrier so that the tracer can be pursued and photographed even in a place where we are obliged to pay attention to our footholds. As shown in Figure.1, the instrument consists of a shooting device (inertial camera) equipped with a camera and an inertial measurement unit (IMU) and a controller equipped with a battery and a personal computer. An eyepiece attachment is set on the camera to make it easy to pursue the tracer, enabling us to look through the viewfinder from above. The camera is a single-lens reflex digital camera Canon EOS1Ds Mark2 DIGITAL (nominal picture distance: $35 \mathrm{~mm}$; screen size: $36 \mathrm{~mm}$ x $24 \mathrm{~mm}$; valid pixels: approx. 16.7 million pixels; total pixels: 17.2 million pixels). IMU is TA7431 made by Tamagawa Seiki Co., Ltd. The angular velocity sensor is a monolithic ring laser gyro T-16B made by Kearfott Corporation, an American manufacturer and with a bias of $+0.3 \mathrm{deg} / \mathrm{h}$. The accelerometer is a high-accuracy accelerometer QA-1200 made by AlliedSignal Inc., an American manufacturer and with a bias of +300 microG. The shutter is operated manually but the IMU displays the signal of shutter and is synchronized with the camera. 


\subsection{Inertial stereo Photogrammetry Instrument}

In this study, an instrument is modified to enable stereo photogrammetry using two cameras, in addition to inertial single photogrammetry, as shown in Figure.2. The camera base length is fixed at $1.2 \mathrm{~m}$, taking into account the operability. The camera is a single-lens reflex digital camera Canon EOS-40D (screen size: $22.2 \mathrm{~mm}$ x $14.8 \mathrm{~mm}$; valid pixels: approx. 10.1 million pixels; total pixels: 10.5 million pixels) whose shutter automatically clicks in a continuous way after power-on via a radio controller (proportional system), enabling sequence photography with intervals of 0.33 seconds. This system allows selection between manual single-photograph and automatic stereo-photograph.

\subsection{Theodolite Camera}

This study adopted a theodolite camera, theodolite equipped with a digital camera, in order to allow calculating the shooting positions and camera inclination, which are exterior orientation parameters. The camera used is the same as that used for the inertial stereo photogrammetry instrument. Four instruments were prepared and all of them are synchronized by radio control to enable sequence photography. The theodolite measures angles with an accuracy of 20 seconds, which allows the photogrammetry requiring no control point. Figure.3 shows the theodolite camera.

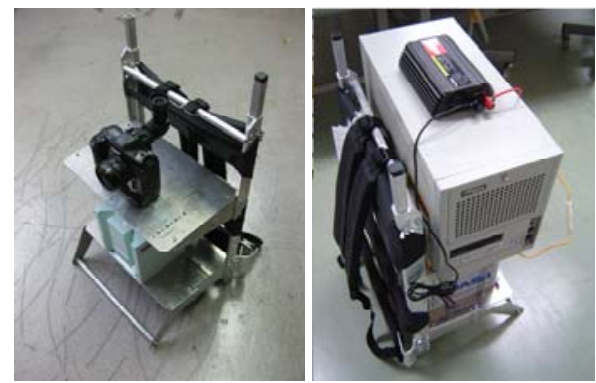

Figure 1. Inertial single photogrammetry instrument

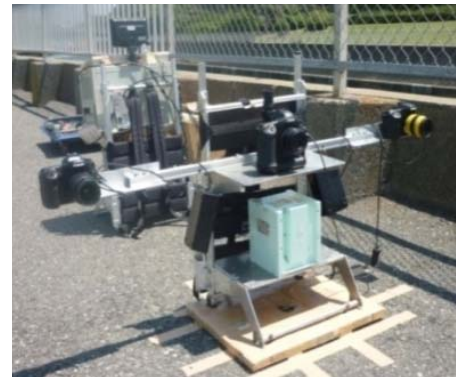

Figure 2. Inertial stereo photogrammetry

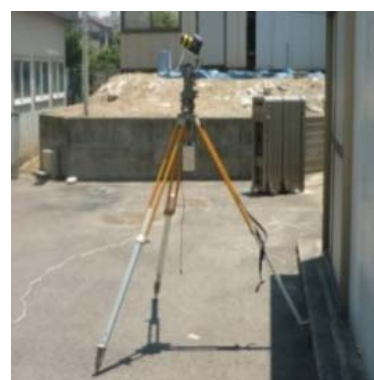

Figure 3. Theodolite camera

\section{TRACER}

\subsection{Spherical rubber balloon}

A spherical rubber balloon, which is used as meteorological balloon, was adopted as tracer and was filled with mixture of helium gas and air to be the same weight as the air. Its diameter is fixed to $40 \mathrm{~cm}$ because this diameter ensures operability and allows identification even $50 \mathrm{~m}$ away from the camera. Figure.4 shows the balloon used.

\subsection{Soap Bubbles}

Unlike balloons, soap bubbles can be released in large quantity within a short duration, so we can expect they allows more detailed observation of wind flow than balloons. The purpose of this study is to develop a soap bubble generator that can continuously release bubbles of a diameter of $40 \mathrm{~cm}$.

At this stage, the best mixture of soap solution is composed of water, granulated sugar, starch and dishwasher detergent in a ratio of 10:10:5:1 by weight. Figure.5 shows the soap bubble generator.

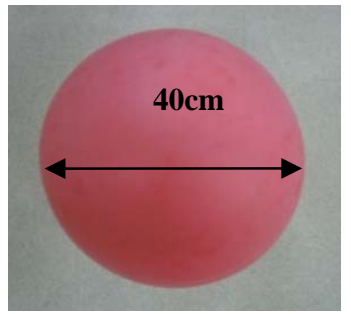

Figure 4. Spherical Rubber Balloon

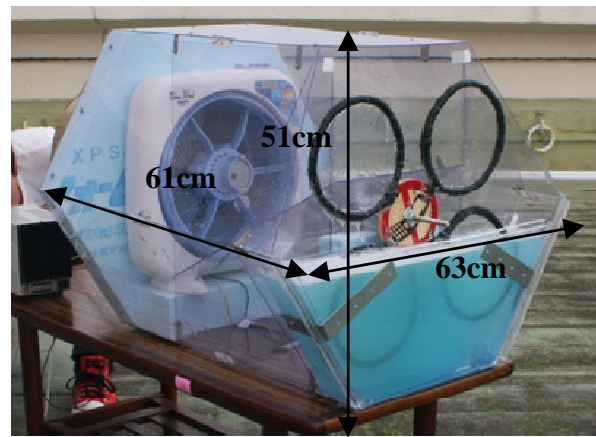

Figure 5. Soap Bubble Generator

\section{EXPERIMENT OF OBSERVATION ACCURACY}

The purpose of experiment is to verify the observation accuracy of the inertial single photogrammetry instrument, inertial stereo photogrammetry instrument and theodolite cameras described in Chapter 2.

\subsection{Test Method}

The experiment was carried out in an open park. Figure. 6 shows a view of experiment. A tricycle loaded with a VRS-GPS and a tracer (balloon) ran at a speed of about $5 \mathrm{~m} / \mathrm{s}$ and was photographed by the inertial single photogrammetry instrument, inertial stereo photogrammetry instrument and theodolite cameras. The photographs thus taken were used for analysis to calculate the three-dimensional position of the tracer and to draw its trajectory. Additionally, the position data were obtained also by VRS-GPS on the tricycle and used for calculating the tracer's trajectory. The position data obtained by 
VRS-GPS were treated as true values, which were compared with the results of observation methods to verify the accuracy. It should be noted that VRS-GPS and other observation methods were synchronized by manually pressing switches so they were not deemed as being precisely synchronized.

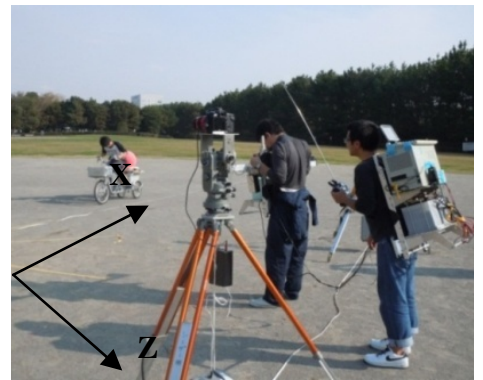

Figure 6. View of Experiment

\subsection{Result of Experiment}

The accuracy was verified by comparing the drawn trajectories. Among the trajectories obtained by each method, one whose measurement time is the closest to that of VRS-GPS was adopted. It is because the synchronization is not precise. Figure.7 shows the result of experiment. According to this experiment, each method turned out to be able to provide a trajectory almost equal to one of VRS-GPS. Figure.7 proves that the data from about $65 \mathrm{~m}$ deviate. This deviation is considered to have occurred because the balloon's apparent size became smaller on photos. From this result, the analysis range of the inertial single photogrammetry instrument with a balloon of a diameter of $40 \mathrm{~cm}$ seems to be about $65 \mathrm{~m}$ from the shooting position.

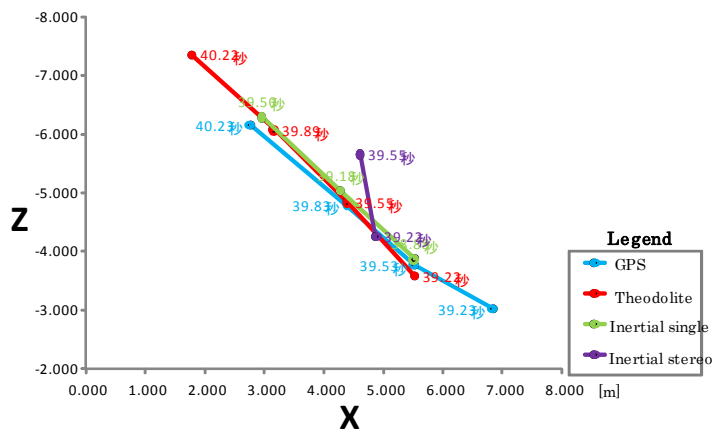

(a) Displacement experiment in proximity

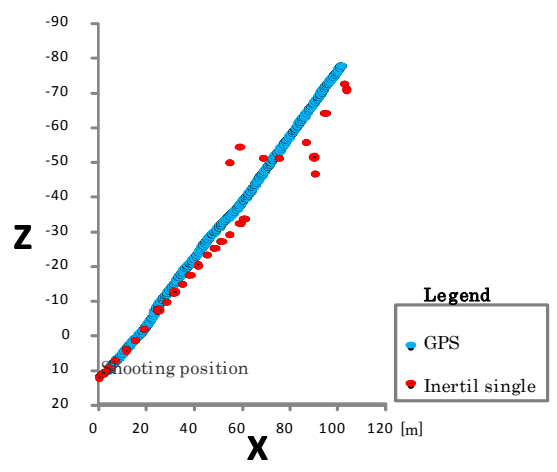

(b) Dsiplacement experiment with a long distance

Figure 7. Comparison between trajectories

\section{EXPERIMENT OF OBSERVATION OF WIND FLOWS AROUND EMBANKMENT}

Paths of particle of winds that flow around embankment were measured in a three-dimensional way.

\subsection{Experiment Method}

The experiment was carried out on a seaside embankment. In this experiment, a soap bubble generator was placed on a tetrapod and bubbles were released from it to make use of winds flowing from the sea to the embankment. A balloon was also released from the same position as the soap bubble generator. The released tracers were photographed by the inertial single photogrammetry instrument, inertial stereo photogrammetry instrument and theodolite camera. The photographs thus taken were used for analysis to calculate the three-dimensional position of the tracers and to draw their paths of particle. Figure. 8 shows the view of experiment.

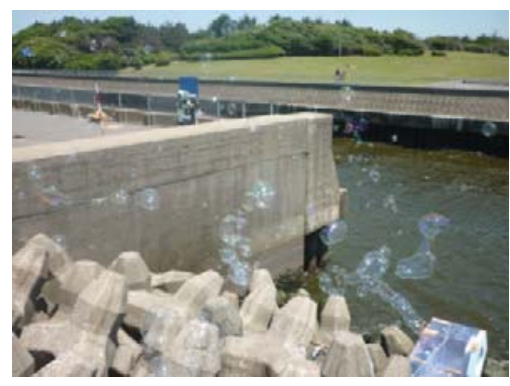

Figure 8. View of Experiment

\subsection{Result of Experiment}

Figure. 9 and 10 show the results of experiment obtained by each of observation methods with released tracers. The red path of particle shows the trajectory of balloon and the blue one shows that of a soap bubble. These figures enable clearly understanding how winds flowing over the embankment go upward above it and go downward behind it. An anemoscope and an anemometer installed there show that the wind was blown from about south (S) at a speed of about $3.46 \mathrm{~m} / \mathrm{s}$, which is almost same as analyzed from the photographs.

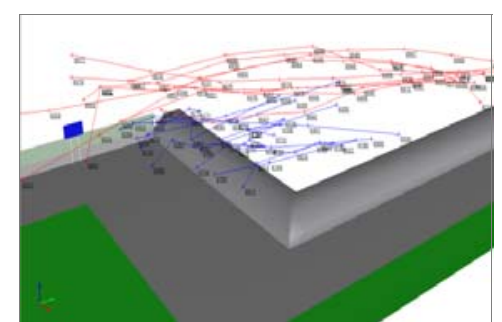

Figure 9. Paths of particle obtained by the Inertial single photogrammetry

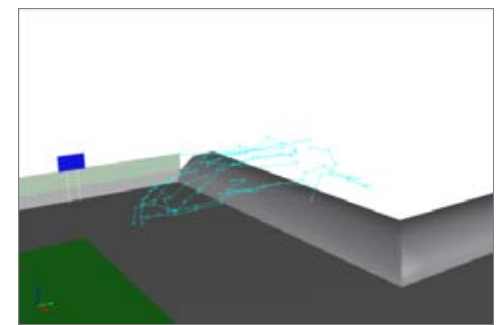

Figure 10. Paths of particle obtained by the Inertial stereo photogrammetry 


\section{EXPERIMENT OF OBSERVATION OF CONICAL VORTEX AT A CORNER OF BUILDING}

The purpose of this experiment is to observe conical vortexes generated at a corner of building in a three-dimensional way.

\subsection{Experiment Method}

The experiment was carried out on the roof floor of a 9-story reinforced concrete building. It was intended for observing conical vortexes generated by winds hitting the corner of a building. Figure.11 shows the appearance of the building. Four theodolite cameras were installed at intervals that enable stereo photography and a soap bubble generator was set on the windward side. The photos thus taken were subjected to photogrammetry to calculate the three-dimensional position of bubbles and to draw their paths of particle.

\subsection{Result of Experiment}

Figure.12 shows the paths of particle obtained from the experiment. These lines clearly prove that conical vortexes were generated by winds hitting the corner of edge of eaves of the roof floor on the windward side. It was also planned to carry out observation by the inertial single photogrammetry instrument and intertial stereo photogrammetry instrument, but the analysis was given up because too large number of bubbles and their too complicated move made stereo-matching impossible. It turned out that observation by theodolite cameras allowing sequence photography is useful for a situation like this experiment where the wind flows in a complicated way.

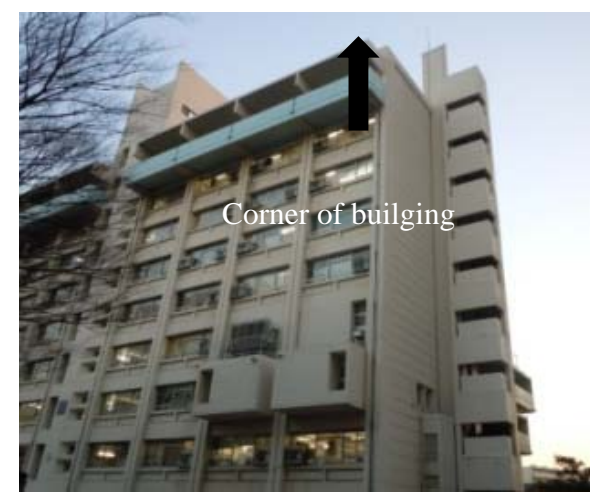

Figure 11. Outer view of building where the observation was carried out

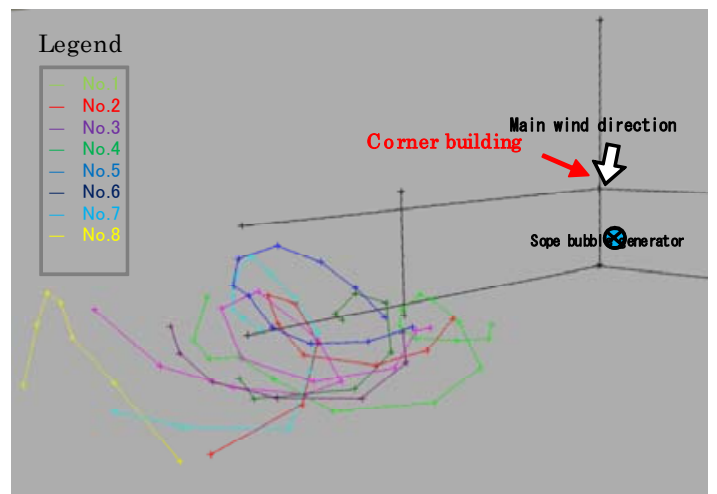

Figure 12. Paths of particle of conical vortex at a corner of building

\section{EXPERIMENT OF OBSERVATION OF WIND FLOWS AROUND A HIGH BUILDING}

The purpose of this experiment is to observe winds flowing around a high building in a three-dimensional way.

\subsection{Experiment Method}

The experiment took a 20-story reinforced concrete building as object. Figure.13 shows the view of experiment. In order to see the wind flows around the high building, tracers were released from the 9th floor level of the outside staircase of a 10-story building situated on the windward side. Tracers released from windward were photographed by the inertial single photogrammetry instrument. The photos thus taken were subjected to analysis to calculate the three-dimensional position of tracers and to draw their paths of particle.

\subsection{Result of Experiment}

Figure.14 shows the result of experiment obtained by an inertial single photography camera with released balloons. This result enables understanding that winds flow around the high building as if they avoid its higher part.

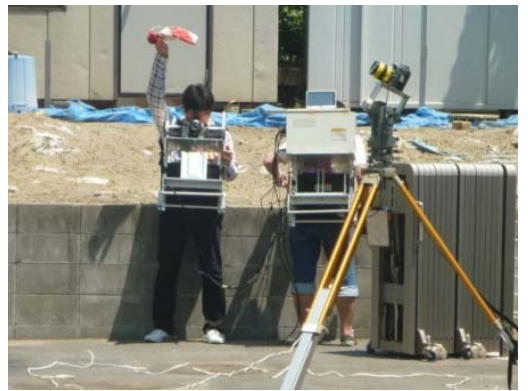

Figure 13. View of experiment

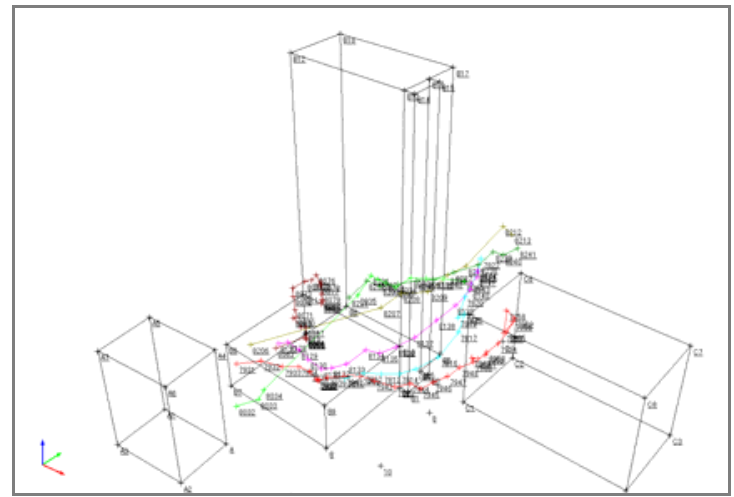

Figure 14. Paths of particle around the high building

\section{CONCLUSION}

(1) We prepared soap bubbles useful as tracers in wind observation by photogrammetry and a soap bubble generator.

(2) We developed an inertial single photogrammetry instrument and an inertial stereo photogrammetry instrument as new observation methods.

(3) We summarized the advantages and disadvantages of these observation methods.

(4) With their commercial use in view, we observed wind flows around an embankment, conical vortex at a corner of building and wind flows around a high building according to these 
International Archives of the Photogrammetry, Remote Sensing and Spatial Information Sciences, Volume XXXIX-B5, 2012 XXII ISPRS Congress, 25 August - 01 September 2012, Melbourne, Australia

methods. As presented in this study as applications, the instruments were proved to be useful for wind observation.

\section{References:}

Koizumi, T., 2010. Development of wind observation system utilizing inertial single photogrammetry, Journal of the Japan Society of Photogrammetry and Remote Sensing, Vol.49, No.1, pp.21-31.

Koizumi, T., et. al., 2011. Development of a wind observation system utilizing simplified aerial photogrammetry (3) -The measurement of wind flow by phototheodolite and soap bubbles-, Journal of Wind Engineering, JAWE, Vol.36, No.1 (No.126), pp.1-12. 